\title{
Multi-dimensional linked-data exploration with
}

\section{glue}

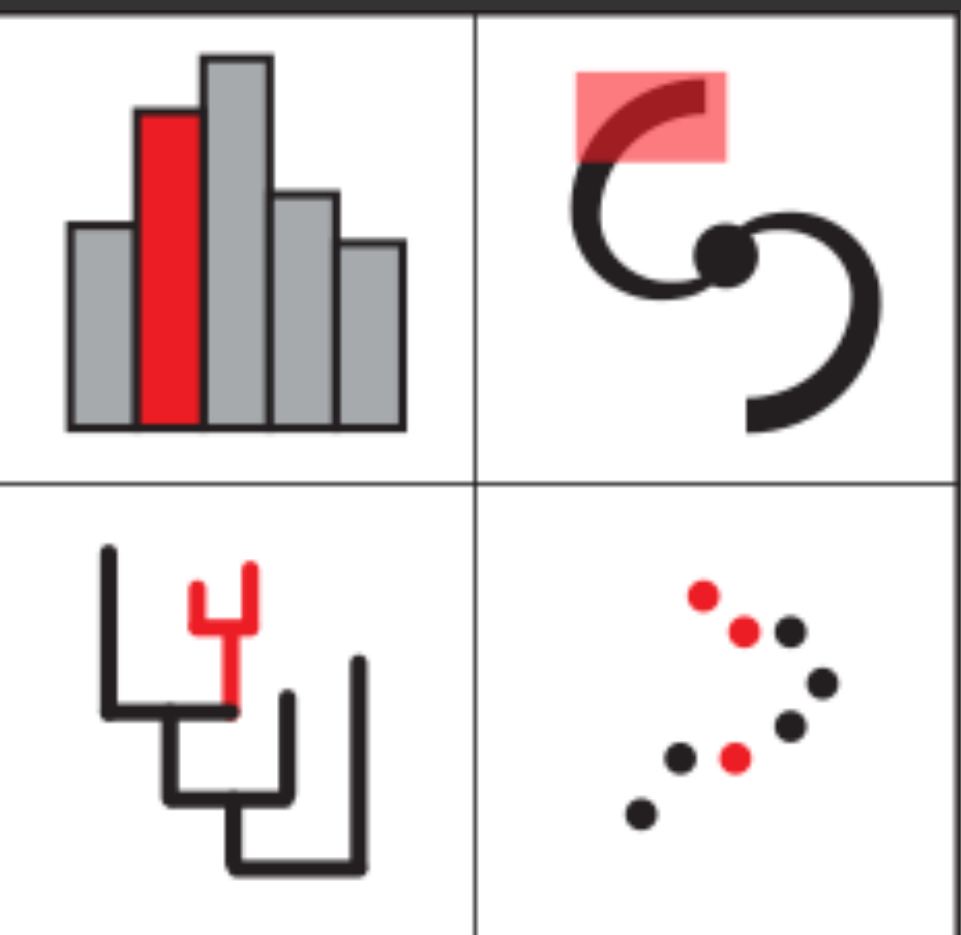

Tom Robitaille

Scientific Software developer @ Aperio Software

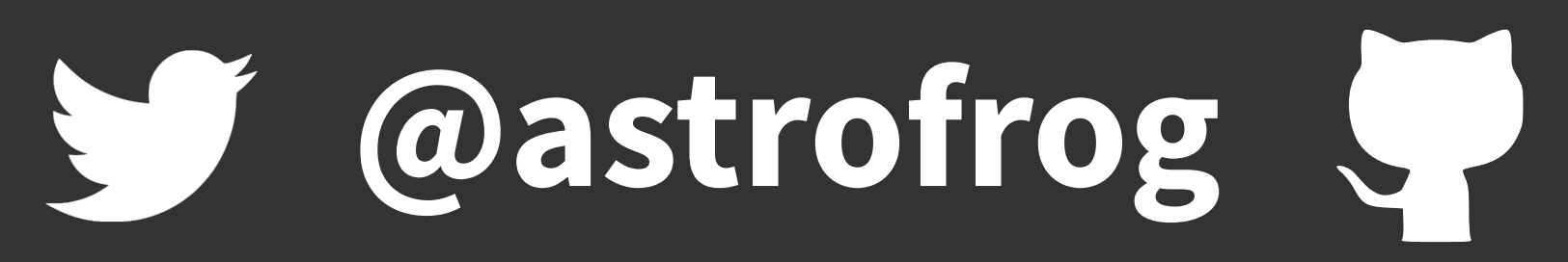

Alyssa Goodman (PI), Chris Beaumont, Maarten Breddels,

Catherine Zucker, Hope Chen, Michelle Borkin 


\section{glue is a multi-disciplinary Python package for multi-dimensional and linked data exploration}




\section{Data can be highly-dimensional}

Need linked interactive visualizations

\section{Data can be heterogeneous}

(multiple files, varying dimensionality, conventions, etc.)

Need linking of data

\section{Often interested in a subset of the data}

Need a way of constructing/combining selections 


\section{Written in Python, and has both a Qt and now a Jupyter user interface}



Python
File Edit View
Canvas Data Manager Plugins Help

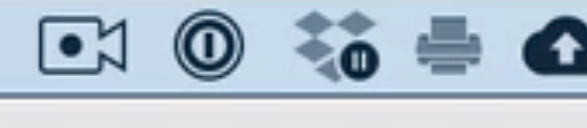

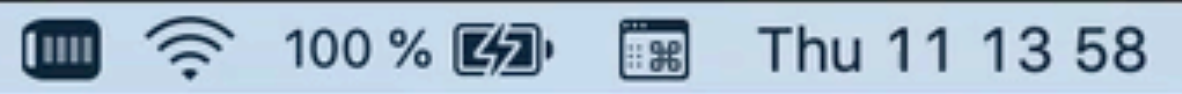
Open Session 回 Export Session
Import Data
圆 Export Data/Subsets L Link Data $x^{2}$ Arithmetic attributes
Active Subset: None/Create New $\times$ Tab 1
IP. Terminal \$Preferences Data Collection

Data

Subsets

Plot Options

\section{Drag Data To Plot}




\section{Auto-linking of $n$-dimensional datasets and on-the-fly reprojection}



Python
File Edit View Canvas Data Manager Plugins Help

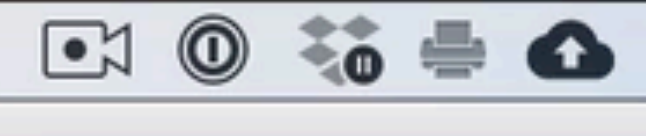

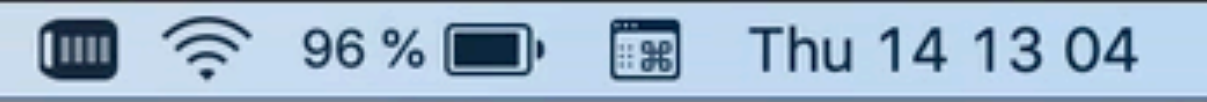
Open Session
Import Data Active Subset: $N$ None/Create New $\quad \hat{v}$ (the next selection will create a subset)
IP. Terminal
* Preferences Data Collectio $\times$ Tab 1

Data

Subsets

Drag Data To Plot

Plot Options

Plot Layers 


\section{Support for large/remote/unstructured datasets}

[demo data with 146 millon rows from http://vaex.astro.rug.nl/latest/datasets.html] 
Open Session Export Session Import Data $\quad$ Export Data/Subsets $\quad$ Link Data $x^{2}$ Arithmetic attributes Active Subset: $N o n e / C r e a t e ~ N e w \quad \hat{v}$ (the next selection will create a subset)

$$
\text { Data }
$$

nyc_taxi_2015

Subsets

\section{Plot Layers - 2D Scatter}

$\checkmark$ nyc_taxi_2015

\begin{tabular}{l|l|l|l|l} 
Color & Points & Line & Errors & Vectors \\
\hline
\end{tabular}

color Fixed $\hat{v}$

opacity

Plot Options - 2D Scatter

General Limits Axes

x axis pickup_longitude

$\hat{\imath} \log$

y axis pickup_latitude

$\hat{v} \log$

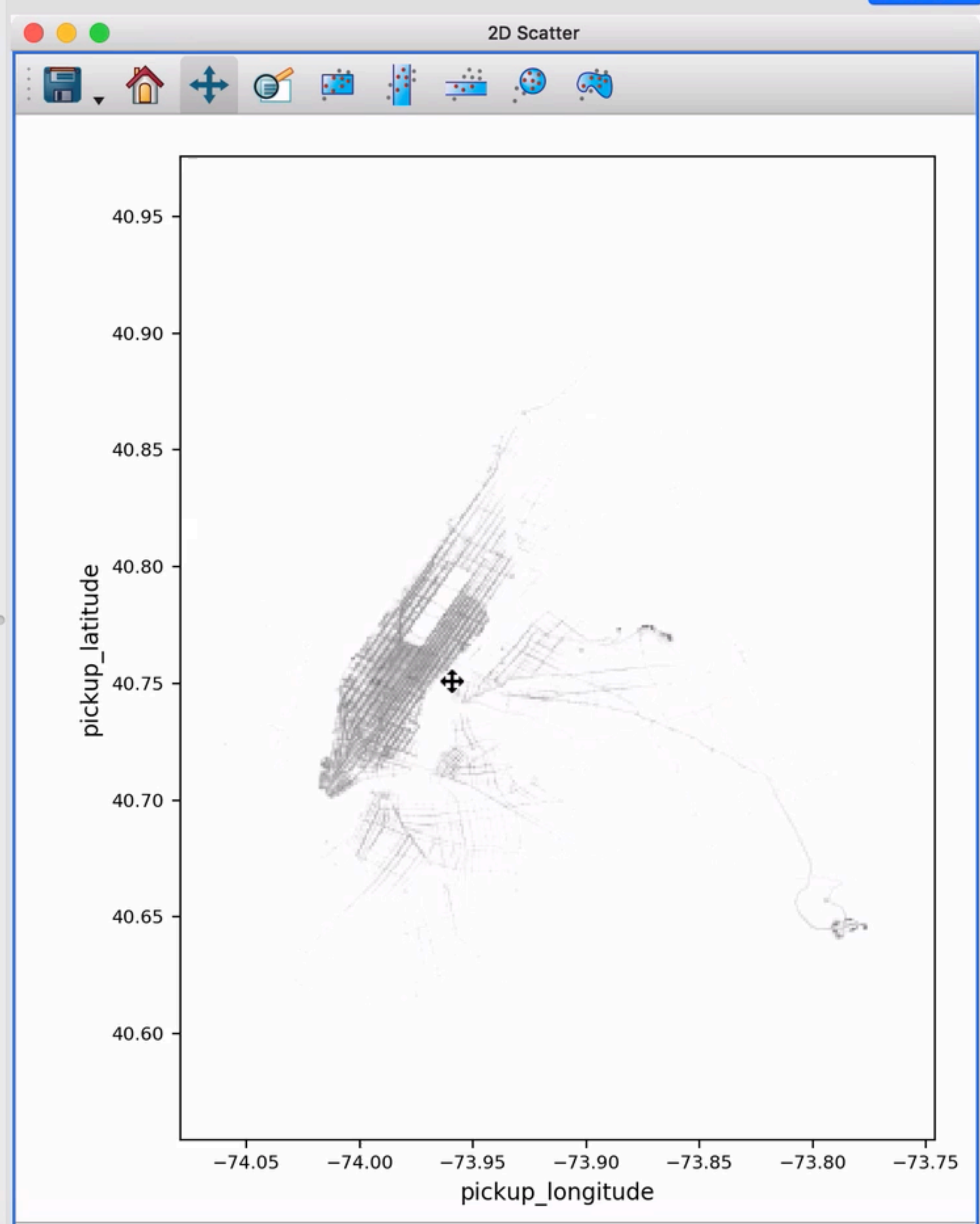

2D Scatter

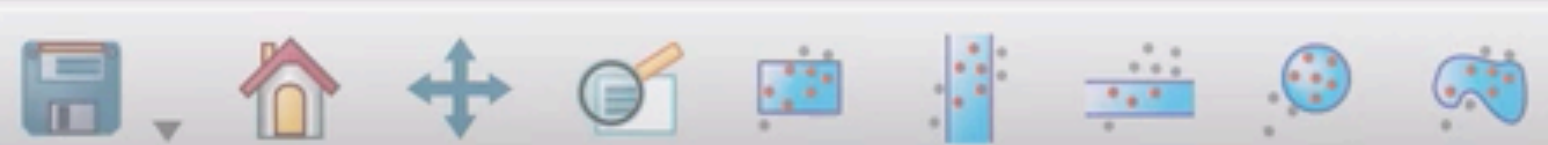

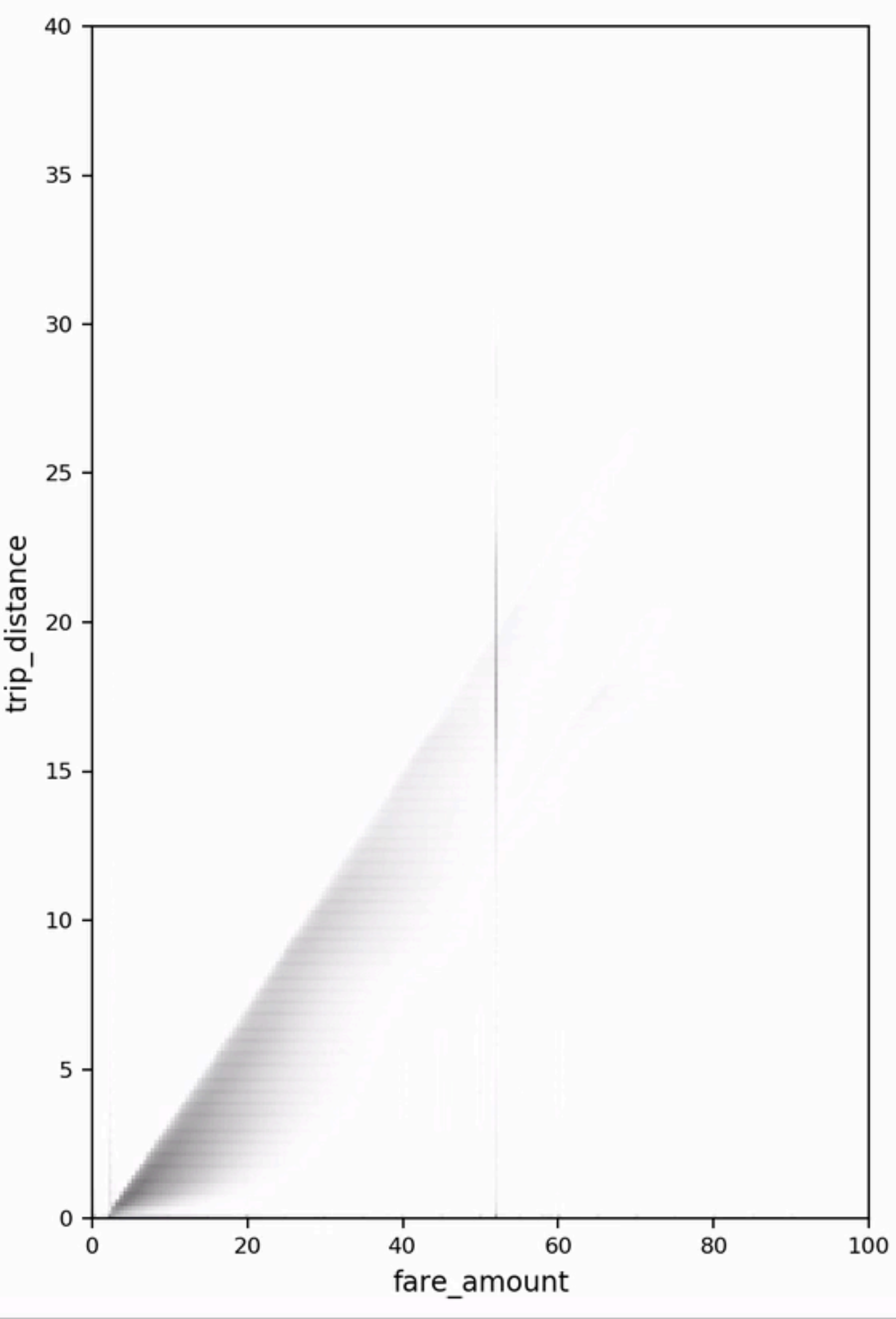


Highly customizable/extensible 
from glue.config import data_factory

from glue.core import Data

from skimage.io import imread

def is_jpeg(filename, **kwargs):

return filename.endswith('.jpeg')

@data_factory('JPEG image loader', is_jpeg)

def read_jpeg(filename):

im = imread (filename)

return Data(cube=im) 
from glue.config import link_function

@link_function(info="Link from deg to rad", output_labels=[ 'rad' ])

def deg_to_rad(deg):

return deg * $3.14159 / 180$ 


\section{Extensible with plugin packages}




\section{glue-geospatial}

Support for geospatial file formats https://github.com/glue-viz/glue-geospatial

\section{glue-medical}

Support for medical file formats (e.g. DICOM) http://github.com/glue-viz/glue-medical 


\section{glue-wwt}

Embedding of WorldWideTelescope in glue http://github.com/glue-viz/glue-wwt

\section{glue-plotly}

Support for exporting plots from glue to plot.ly https://github.com/glue-viz/glue-plotly 
á Python File Edit View Canvas Data Manager Plugins Help

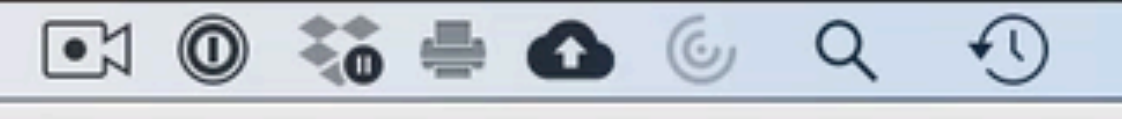

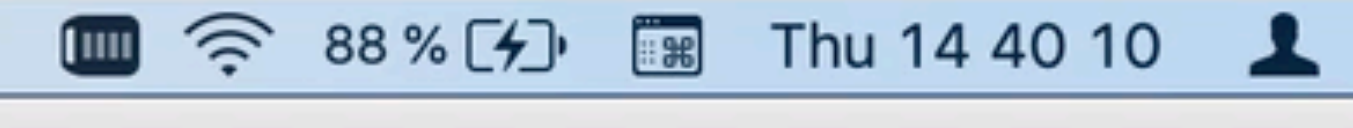

: Open Session 圆 Export Session Import Data 圆 Export Data/Subsets Link Data $^{2}$ Arithmetic attributes Active Subset: $N o n e / C r e a t e ~ N e w \quad \hat{v}$ (the next selection will create a subset)

IP. Terminal

\$ Preferences Data Collection

Data earthquakes_2010

Subsets

Plot Layers - WorldWideTelescope (WWT)

$\checkmark$ earthquakes_2010

\section{Size $\quad$ Color}

Fixed $\hat{\imath} 2$

Plot Options - WorldWideTelescope (WWT)

\begin{tabular}{r|l|r|} 
Mode: & Earth & $\hat{v}$ \\
\cline { 2 - 3 } Longitude & longitude & $\hat{v}$ \\
Latitude & latitude & $\hat{v}$ \\
\hline \multirow{2}{*}{\begin{tabular}{ll} 
depth \\
\hline
\end{tabular}} & $\mathrm{km}$ & $\hat{v}$ \\
\hline
\end{tabular}
$\times$ Tab 1

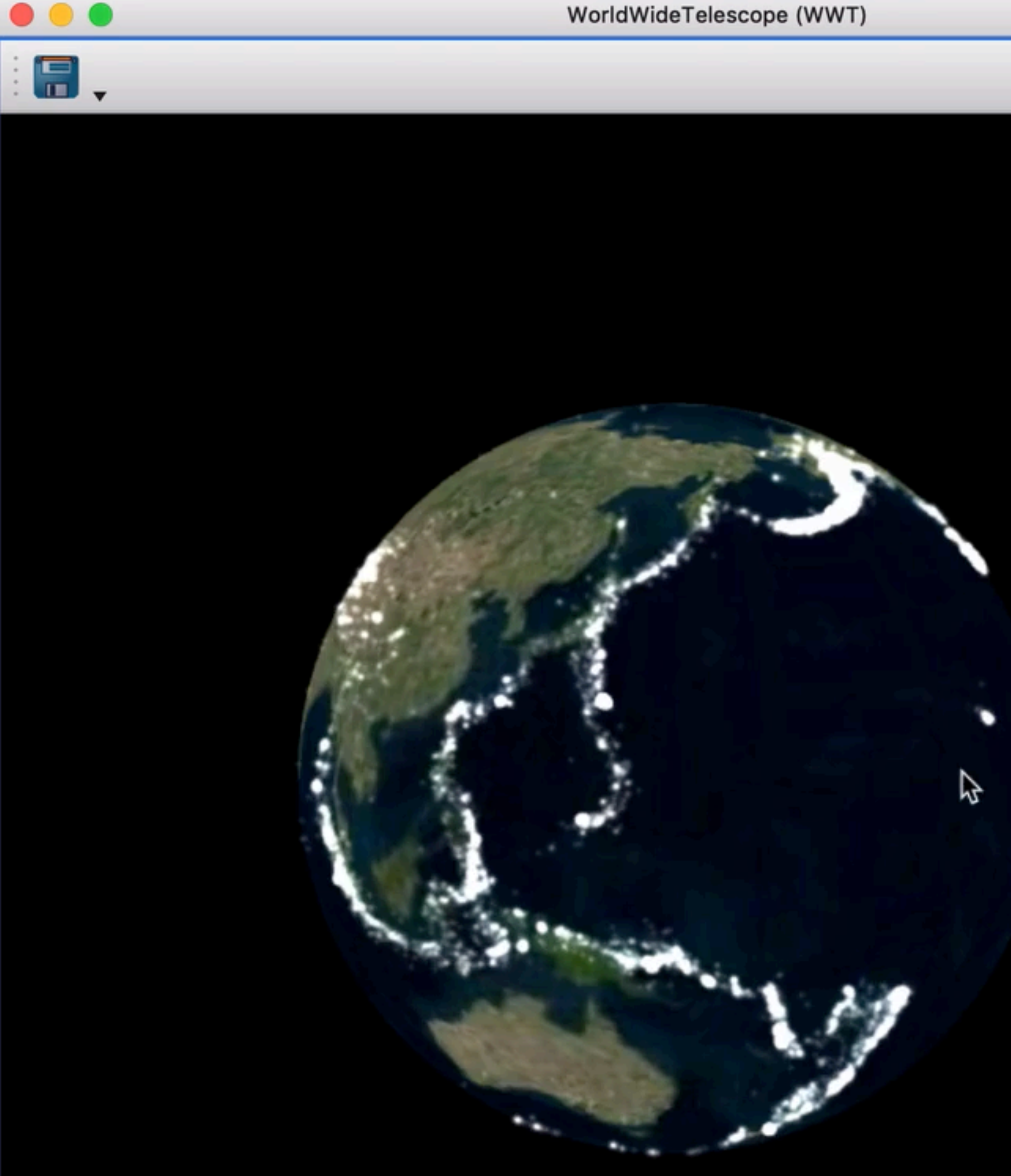

2D Scatter

圆、嵒出

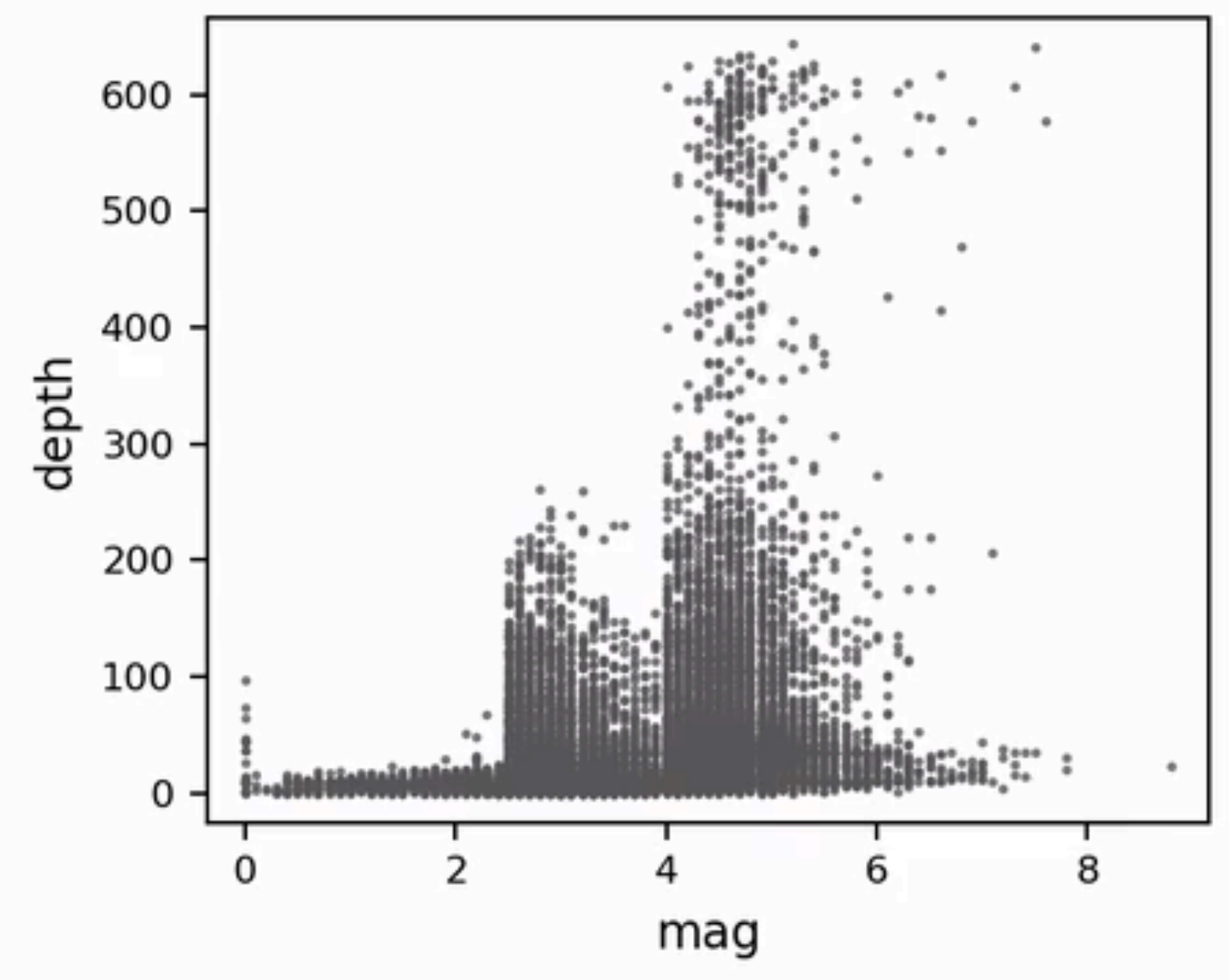

1D Histogram

回, 管 4 ○

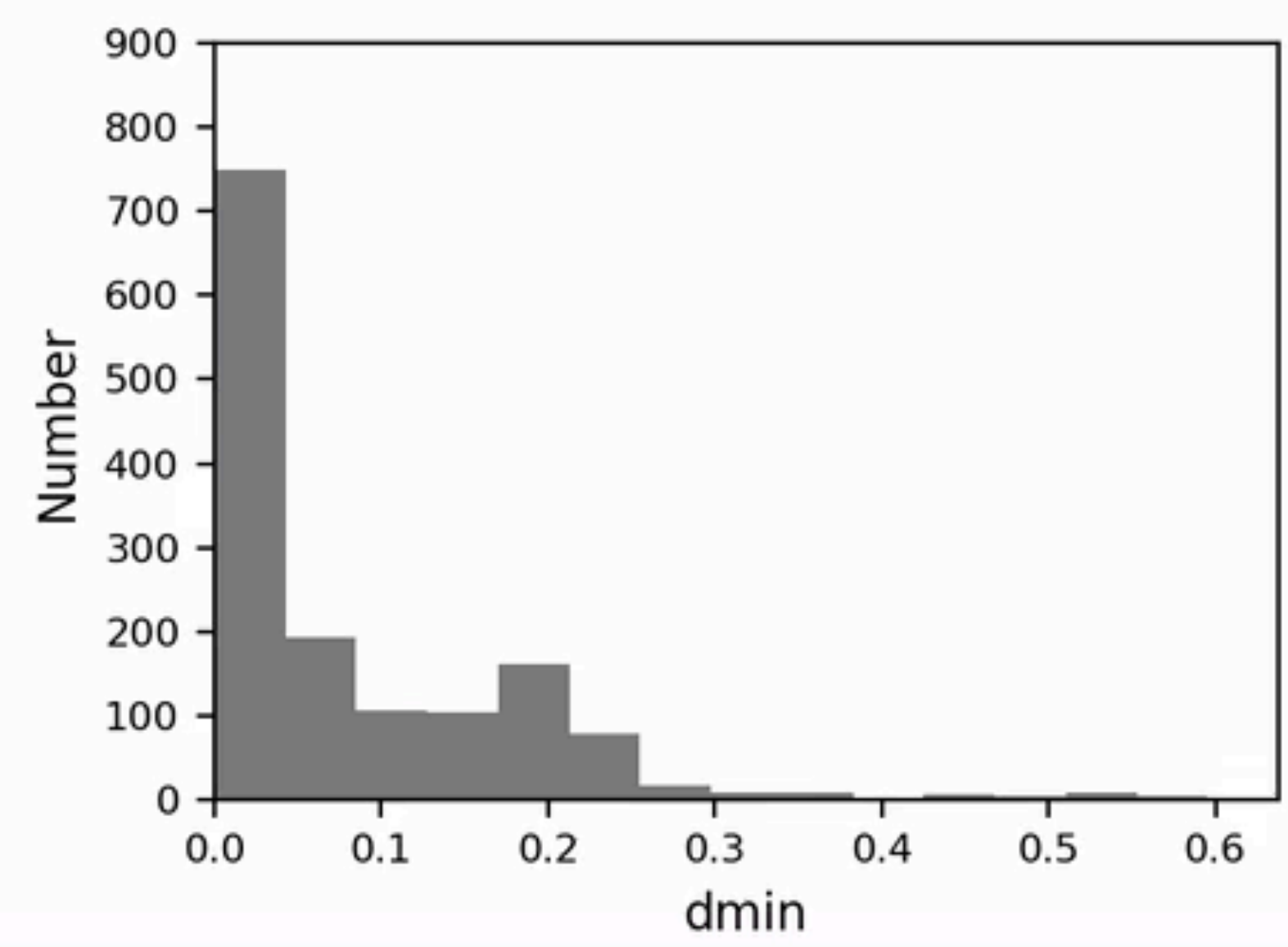


af Python File Edit View Canvas Data Manager Plugins Help

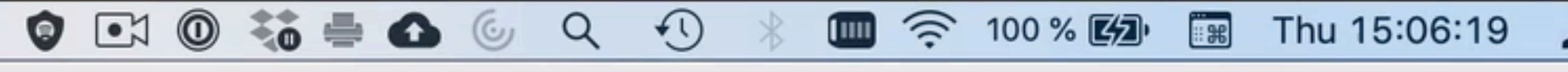

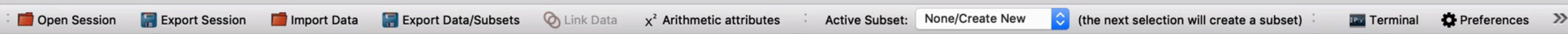
Data Collection

Data
Subsets

\section{Plot Layers - 1D Histogram}

$\checkmark$ NASA exoplanet archive

color

opacity

Plot Options - 1D Histogram

General Limits Axes

x label: Stellar Radial Velocity

$y$ label: Number

axis label size $10 \quad \hat{v} 10 \quad \hat{v}$ axis label weight normal $\hat{v}$ normal $\hat{v}$

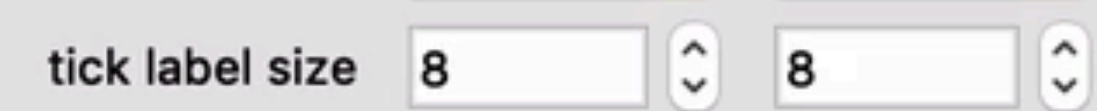

Apply to all plots

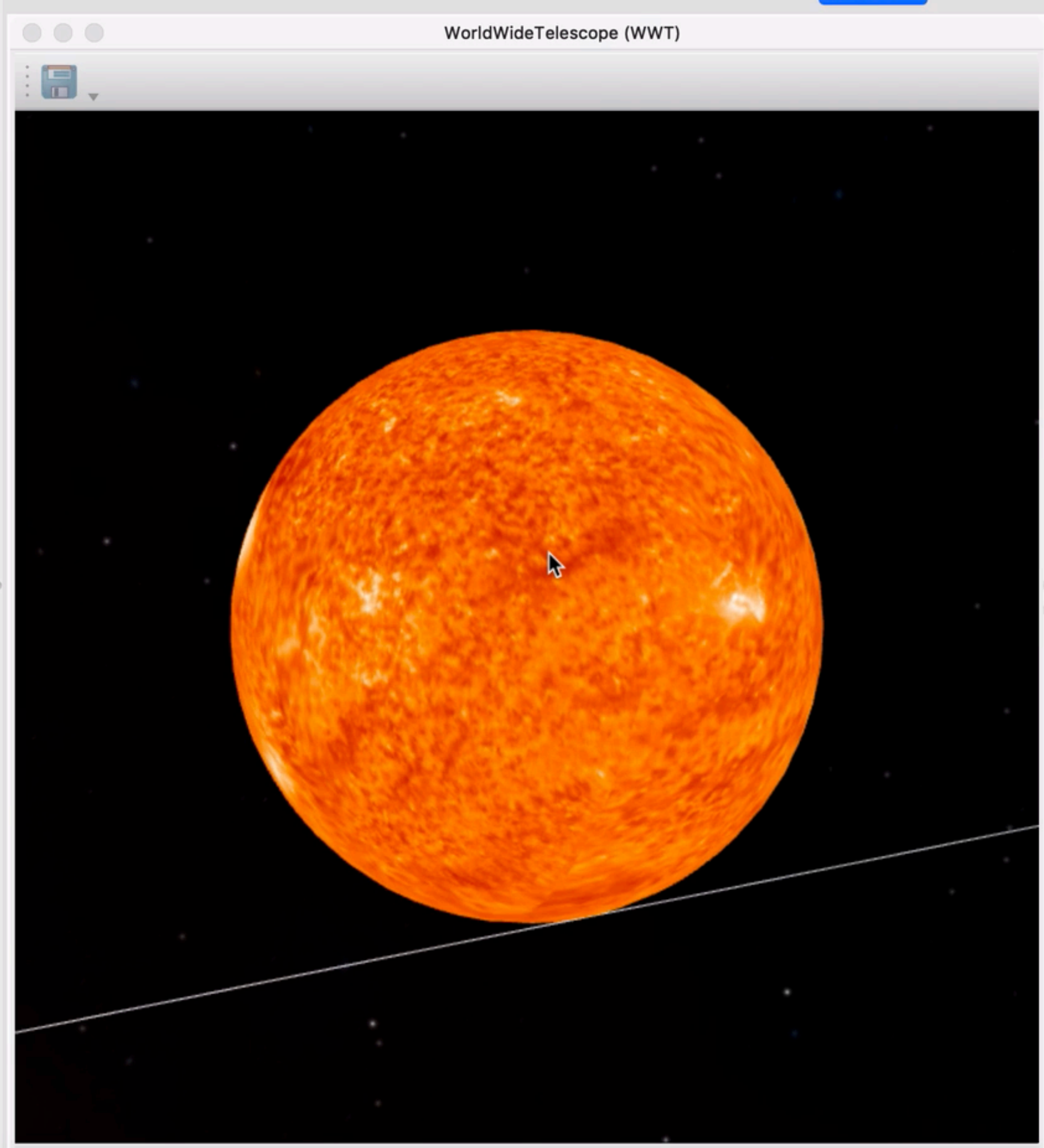

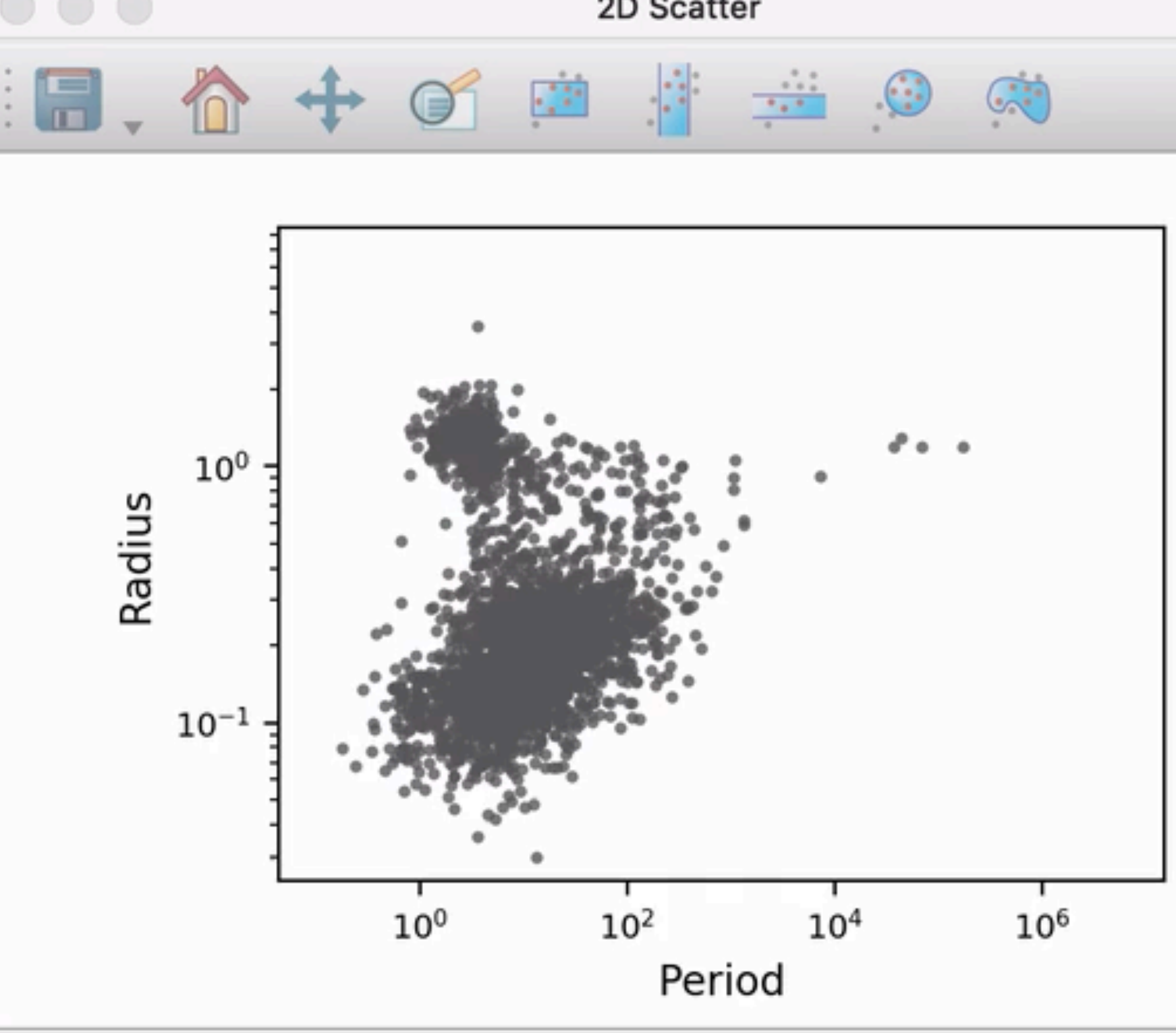

00

圆、合士0 胡

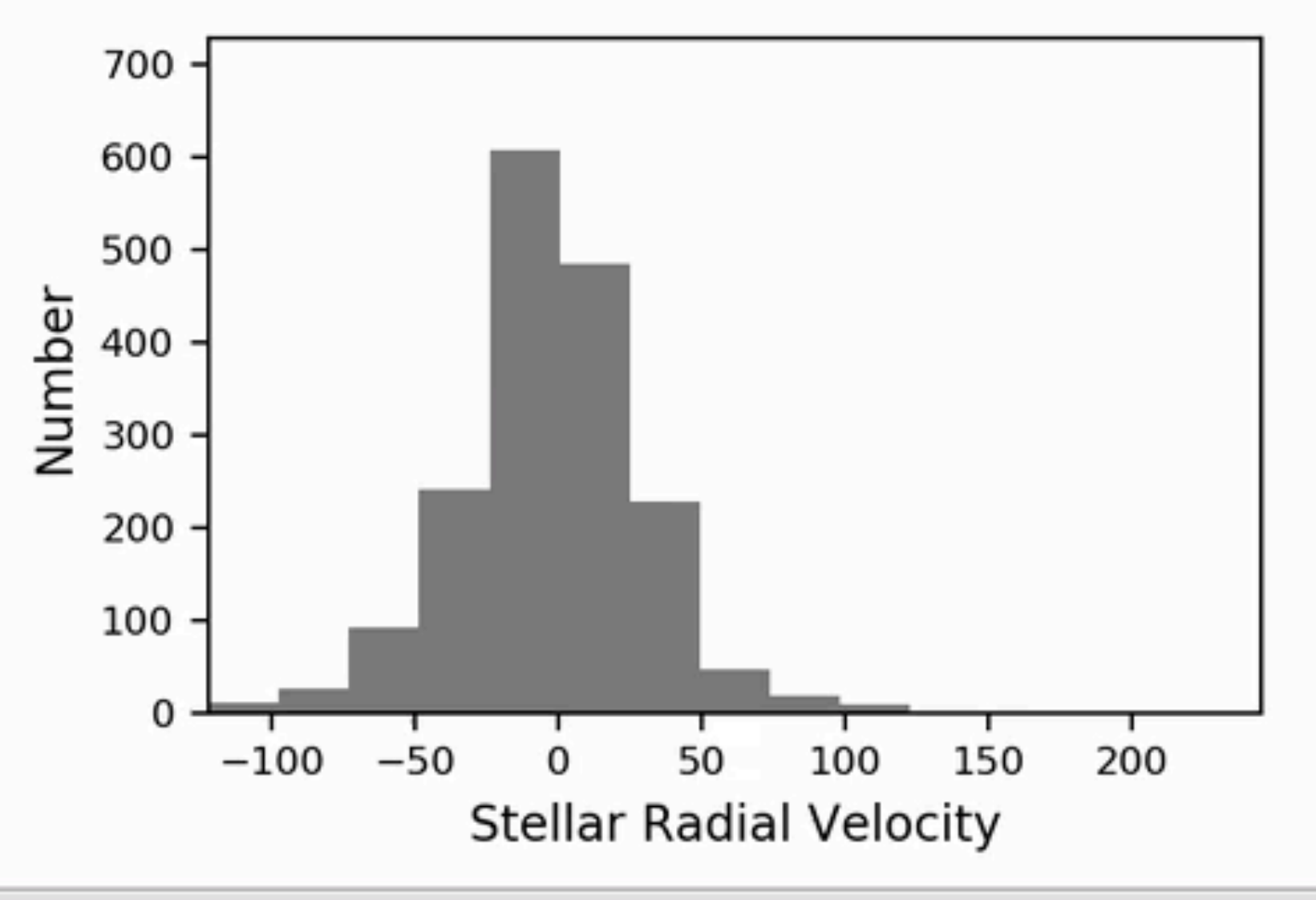


á Python File Edit View Canvas Data Manager Plugins Help

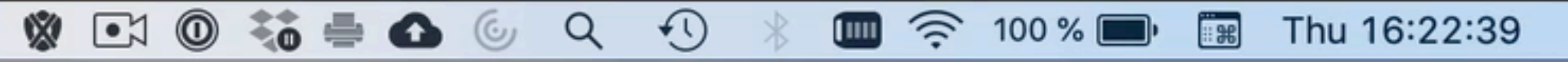

2 : $\equiv$

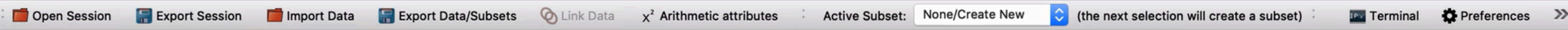
Data Collection

Data

planets_2019.07.11_12.50.45

Subsets

2D Scatter

3D Scatter

\section{Plot Layers - 2D Scatter}

$\checkmark$ | I planets_2019.07.11_12.50.45

\begin{tabular}{l|l|l|l} 
Color Points Line Errors Vectors \\
\hline
\end{tabular}

color Linear $\hat{v}$

attribute st_teff $\hat{v}$

limits 575 $\rightleftarrows 15000$

colormap 100 $\times$ Tab 1

opacity

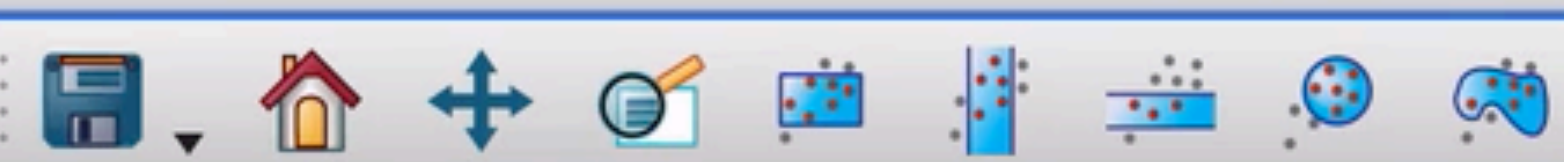

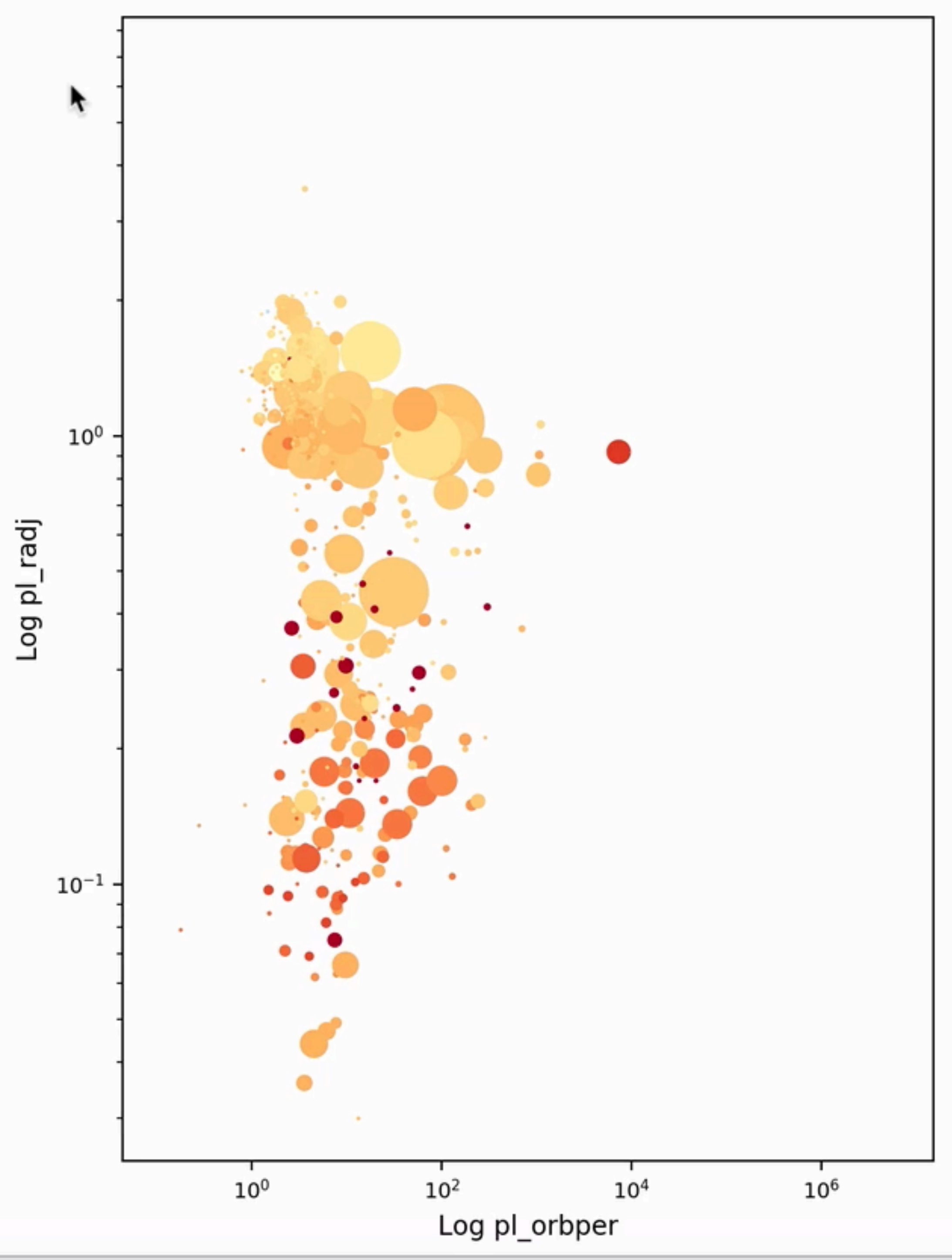

圆, 管口

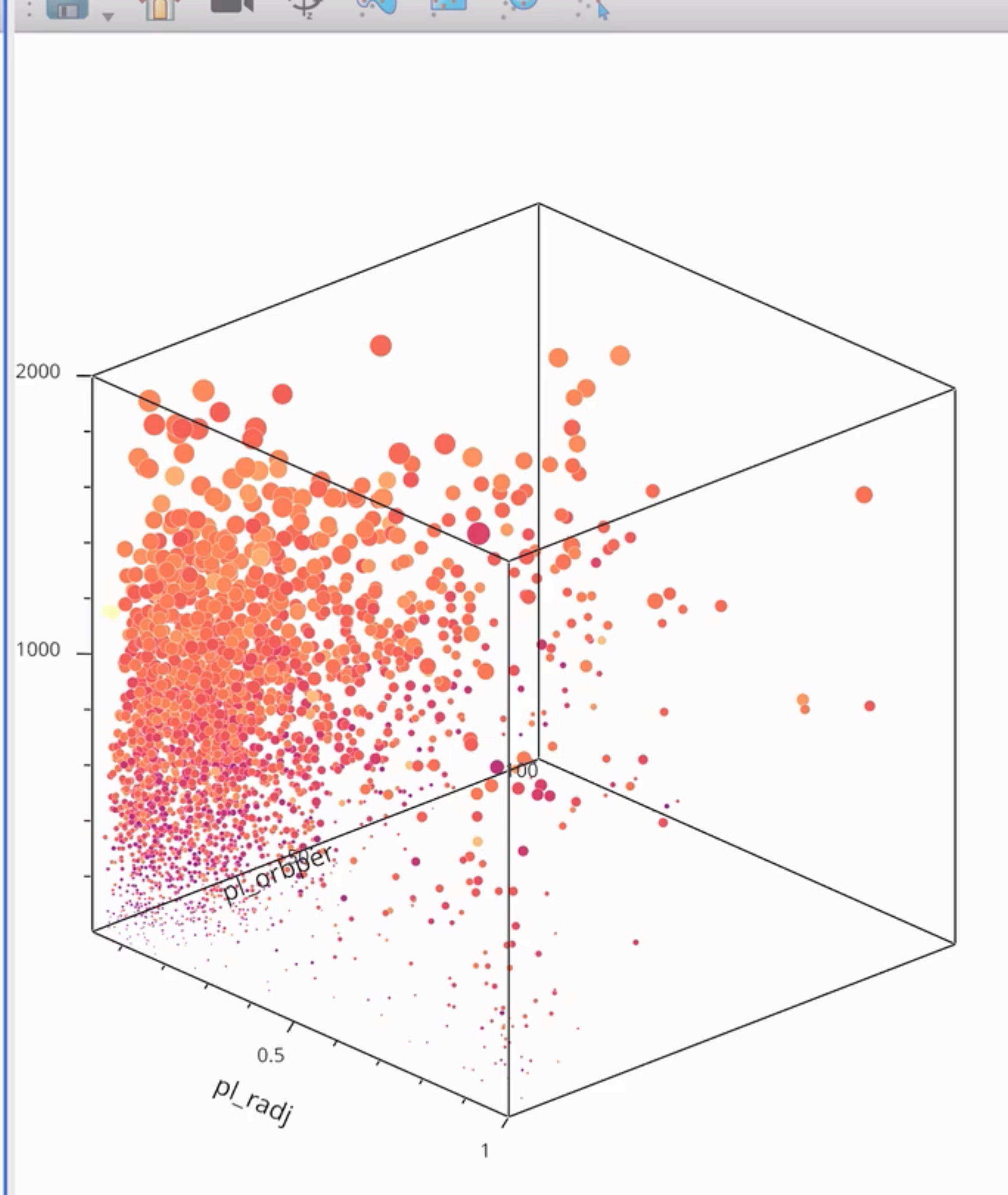

lot Options - 2D Scatter

General Limits Axes

x axis pl_orbper

₹ $\log$

$y$ axis pl_radj $\hat{v} \log$ 


\section{glue-plugin-template}

An empty plugin package to help you get started! https://github.com/glue-viz/glue-plugin-template 


\section{glue in Jupyter notebook and Lab}

\section{"glupyter"}

Much of glue is front-end independent

Relies on other widget libraries, e.g. bqplot

Still at prototype phase 


\section{Snakes on a Plane (well, Planes in Python)}

This notebook explores a CSV files that contains the position, speeds and other related information for planes in the Boston area over a period of 6 hours.

\section{About the data}

These data were collected by directly recordin' publicly available Automatic dependent surveillance - broadcast (ADS-B) transmissions from planes from a single location (hence the data should not be considered complete). The data can be found in https://github.com/glue-viz/glue-example-data/tree/master/Planes/.

For convenience we can use the require_data function to automatically download them here:

In [ ]: from glue_jupyter.data import require_data

require_data('Planes/boston_planes_6h.csv')

\section{Starting up the glue Jupyter application}

Let's start up glue:

In [ ]: import glue jupyter as gj app $=$ gj.jglue ()

and load in the data: 


\section{Compatible with Python 2.7 and 3.5+}

(0.15 is last major Python 2-compatible release)

Works on Mac/Linux/Windows

BSD Licensed 

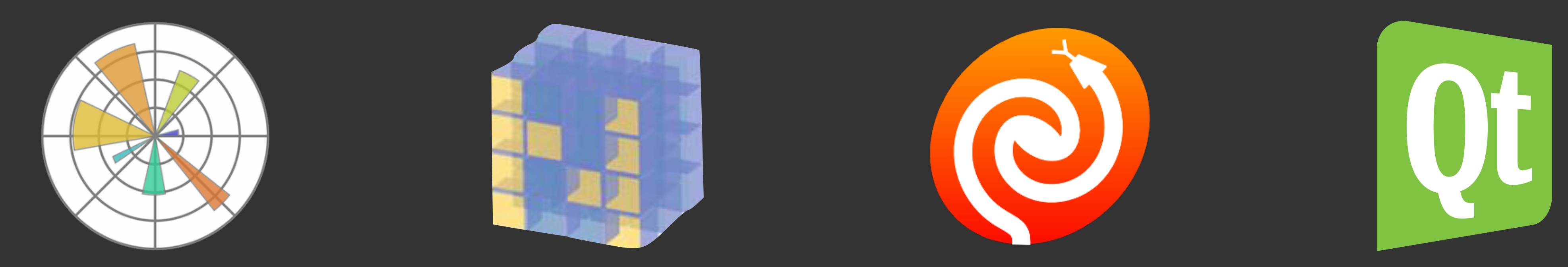

I. VisPy
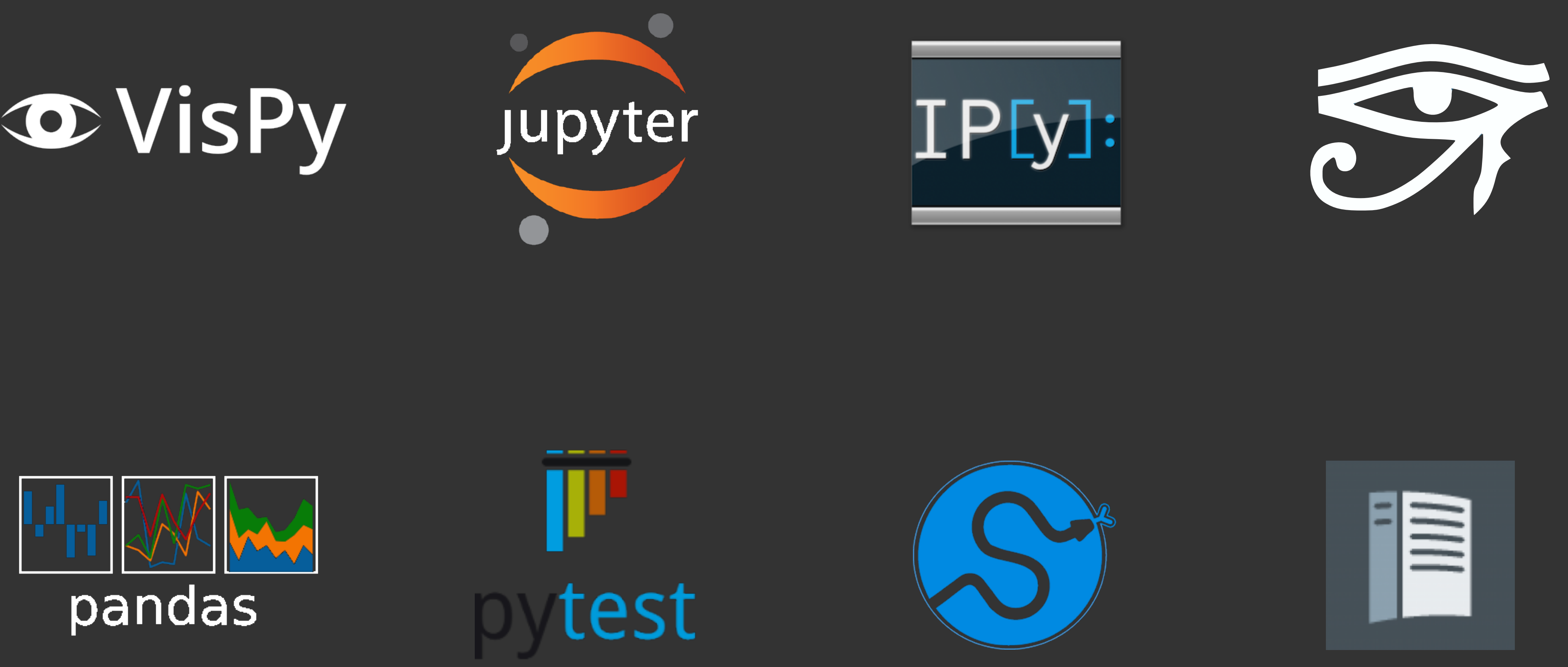

and more! 


\section{http://glueviz.org}

(Tttps://github.com/glue-viz/glue https://github.com/glue-viz/glue-jupyter

@glueviz

Join mailing list and/or Slack! See http://tinyurl.com/glue-help for details 